\title{
El trabajo de la UNESCO sobre la educación de personas adultas y la educación como bien común: Aportes $y$ reflexiones
}

Marcella Milana

\section{Resumen:}

\begin{abstract}
La educación de personas adultas se ha desarrollado en el tiempo como un área particular de investigación, reflexión y acción educativa; un área que favorece una visión de la educación como acción social. A la luz de las múltiples crisis del mundo contemporáneo, este articulo reflexiona sobre el papel complementario que la educación de personas adultas aporta para reforzar el valor educativo de la acción social para el desarrollo de las sociedades contemporáneas a partir de las aportaciones que nos ofrece la labor normativa así como el trabajo conceptual sobre la educación de personas adultas y la educación como bien común, que ha realizado la Organización de las Naciones Unidas para la Educación, la Ciencia y la Cultura (UNESCO).
\end{abstract}

acción; social; desarrollo; educación de personas adultas; educación como bien común; UNESCO. 


\title{
UNESCO's work on adult education and education as a common good: Con- tributions and reflections
}

\begin{abstract}
Adult education has been developed over time as a particular area of research, reflection and educational action; an area that favours a vision of education as a social action. In the light of the multiple crises of the contemporary world, this article reflects on the complementary role that adult education contributes to reinforce the educational value of social action for the development of contemporary societies. This development comes from the contributions that normative work offers us as well as the conceptual work on adult education and education as a common good, which has been carried out by the United Nations Educational, Scientific and Cultural Organization (UNESCO)
\end{abstract}

Keywords: social action; development; adult education; education as a common good; UNESCO.

\section{O trabalho da UNESCO na educação de adultos e na educação como bem comum: contribuições e reflexões}

Resumo: A educação de adultos desenvolveu-se ao longo do tempo como uma área particular de investigação, reflexão e ação educativa. Apresenta-se como uma área que favorece uma visão de educação como ação social. Na sequência das múltiplas crises do mundo contemporâneo, este artigo integra uma reflexão sobre o papel complementar que a educação de adultos carrega para reforçar o valor educativo da ação social par ao desenvolvimento das sociedades contemporâneas, a partir dos contributos legislativos, assim como os contributos teóricos no âmbito da educação de adultos e da educação como bem comum que tem levado a cabo a Organização das Nações Unidas para a Educação, Ciência e Cultura (UNESCO).

Palavras-chave: ação social; desenvolvimento; educação de adultos; educação como bem comum; UNESCO

\section{Le travail de I'UNESCO sur l'éducation des adultes et l'éducation comme bien commun: contributions et réflexions}

Résumé : L'éducation des adultes s'est développé au fil du temps comme un domaine particulier de recherche, réflexion et action éducative. C'est un domaine qui favorise une vision de l'éducation comme action social. A partir des multiples crises que le monde contemporain a vécu récemment, cet article discute le rôle complémentaire de l'éducation des adultes pour le renforcement de la valeur éducative de l'action sociale et pour le développent des sociétés contemporaines. Cette discussion est faite a partir des discussions que nous offrent les documents légaux, mais aussi les propositions conceptuelles, sur l'éducation des adultes et l'éducation comme bien commun développé par l'Organisation des Nations Unies pour l'éducation, la science et la culture (UNESCO).

Mots-clé: action sociale; développement; éducation d'adultes; éducation comme bien commun; UNESCO 


\section{Introducción}

La educación de personas adultas (en adelante EPA) ${ }^{1}$, surgida como una alternativa a los modelos educativos dominantes, se ha desarrollado en el tiempo como área particular de investigación, de reflexión y de acción educativa; un área que favorece una visión de la educación como acción social. Por lo tanto, su papel ha sido interpretado, en el tiempo así como en la geografía europea y mundial, de manera diferente ya sea compensatorio, alternativo o complementario a los modelos dominantes de educación y desarrollo social.

Recientemente, y desde la inspiración de los trabajos de Paulo Freire y Ettore Gelpi, Licinio Lima ha afirmado "que en tiempos de 'posdemocracia'... la educación de adultos es clave para la comprensión crítica de la crisis y para "educar" la crisis a través de la transformación de las estructuras de poder, la economía y la sociedad" (Lima, 2017, p. 2). Es mi creencia que esta tarea, o sea, la de educar la crisis, es algo compartido entre la EPA y la Pedagogía Social. Es en este sentido que me refiero al papel de la EPA como complementaria a otras formas de educación, en las cuales no solo los más vulnerables sino todos los seres humanos participan.

Por lo tanto, a la luz de las múltiples crisis del mundo contemporáneo, es mi intención contribuir a la reflexión sobre el papel complementario que tiene para reforzar el valor educativo de la acción social para el desarrollo de las sociedades contemporáneas, pero desde una perspectiva particular, o sea a partir de las aportaciones que nos ofrece el trabajo sobre la educación de adultos y la educación como bien común realizado por la Organización de las Naciones Unidas para la Educación, la Ciencia y la Cultura (UNESCO).

Para conseguir mi objetivo es necesario volver previamente la mirada al pasado en el espíritu de Paulo Freire que, en la Pedagogía del Oprimido, nos recordó que “... el mirar hacia atrás no debe ser una forma nostálgica de querer volver sino una mejor manera de conocer lo que esta siendo, para construir mejor el futuro" (Freire 1970, p. 66).

En lo que sigue, voy a comenzar por unas anotaciones sobre la historia de la UNESCO, y comentaré su papel en el gobierno mundial de las políticas educativa, antes de examinar tanto su labor normativa, y en particular la Recomendación sobre el aprendizaje y la educación de adultos de 2015, con el trabajo conceptual que condujo, en el mismo año, al documento Replantear la educación ¿Hacia un bien común mundial? (2015). A pesar de que el trabajo de la UNESCO no está exento de críticas, solo enfatizaré su posición política, no neutral, en apoyo a una visión humanista y de contraste al pensamiento neoliberal en la educación. Es mi intención, en fin, considerar unas implicaciones para el futuro de la EPA, y en particular su necesidades, por un lado, de seguir reflexionando sobre la idea de educación como bien común, a pesar de las críticas que esta concepción también presenta, y por otro, de seguir actuando a favor 
de un desarrollo sustentable que rechace el modelo antropocéntrico de sostenibilidad de matriz economicista.

\section{Anotaciones históricas}

Volver la mirada al pasado nos permite reconocer que los intercambios de conocimientos e ideas sobre la educación entre países no son un fenómeno nuevo. Las concepciones e intervenciones pedagógicas en un cierto territorio han sido a menudo más que el resultado de necesidades, capacidades intelectuales y políticas locales por sí solas. Esto es evidente en aquellos territorios que han estado bajo control extranjero desde la primera colonización hispano-portuguesa hasta la descolonización masiva.

Con la descolonización y la expansión capitalista del siglo veinte, el intercambio de conocimientos e ideas sobre la educación se ha ido gobernando a través de la institucionalización de las relaciones internacionales a nivel mundial. La creación de la UNESCO, entre otras organizaciones internacionales, es un ejemplo en este sentido.

Aún en tiempos de conflictos bélicos, en 1942 el Consejo Británico inició la Conferencia de Ministros de Educación Aliados e invitó a Estados Unidos a unirse a la propuesta de crear una organización internacional para promover la cooperación en educación. Esto condujo en 1946 a la fundación de la UNESCO, dentro la Organización de las Naciones Unidas nacida para restaurar la paz y, posteriormente, garantizar la seguridad internacional.

Establecida para promover la paz y la seguridad mediante la aplicación de la educación, la ciencia y la cultura a la comprensión internacional y el bienestar humano, desde su fundación la UNESCO considera que el analfabetismo es irreconciliable con la dignidad humana, y que la educación, la ciencia y la cultura, favoreciendo la solidaridad entre los pueblos, pueden llevar a la paz mundial - aunque los acontecimientos del mundo contemporáneo nos sigan mostrando sus limitaciones para poder realizar esta tarea.

De acuerdo con sus objetivos, como podemos leer en el primer artículo de su Constitución, desde 1949, la UNESCO ha organizado, entre otras actividades, seis Conferencias Internacionales sobre la Educación de Adultos - también conocidas como CONFINTEAs (UNESCO \& MEC, 2014). Éstas, con sus intervalos de aproximadamente doce años, han ido proporcionando el marco para las normas internacionales en materia de EPA (Knoll 2007) - aunque quizás menos en aquellas regiones del mundo económicamente más ricas, como América del Norte y Europa.

Si en 1949 los principales temas e inquietudes de CONFINTEA emanaron de la declaración por la que se fundó la UNESCO, en 1960 los principales temas estaban ya relacionados con los cambios asociados con la expansión de los medios de comunicación masiva, la industrialización de las economías rurales, la introducción de préstamos 
a largo plazo para la educación por el Banco Mundial, y el establecimiento del Instituto de Educación de la UNESCO. Este, nacido como una fundación bajo el derecho civil alemán en 1951, se ha convertido en 2007 en el Instituto de la UNESCO para el aprendizaje permanente, una agencia UNESCO de pleno derecho.

Sin embargo, fue solo en 1972 que la EPA se convirtió en un objetivo de política más allá del nivel nacional. Dos eventos le dieron empuje: el Programa Mundial Experimental de Alfabetización lanzado por la UNESCO unos años atrás y la publicación de Una Introducción a la Educación Permanente por Paul Lengrand (1970). El primer evento llamó la atención de los Estados miembros de las Naciones Unidas sobre la necesidad de erradicar el analfabetismo en todo el mundo, y que la responsabilidad sobre esto recaía en los gobiernos nacionales; el segundo, por otro lado, destacó la importancia, las dimensiones y los objetivos peculiares de la educación permanente, y la necesidad de vincular los procesos de educación aplicados a niños, adolescentes y adultos.

En los años siguientes, cuando las preocupaciones económicas, el pensamiento neoliberal y la teoría del capital humano ya habían encontrado apoyo en todo el mundo, la cuarta CONFINTEA celebrada en 1985 se concentró también en el papel económico de la EPA, e hizo un llamamiento a favor de una mayor cooperación internacional.

Esta preocupación, tuvo su primera concretización en el campo de la educación más allá de la EPA - en 1990, cuando varias organizaciones de las Naciones Unidas encabezadas por la UNESCO, y contando con el apoyo del Banco Mundial, lanzaron el movimiento Educación para Todos.

Esto condujo, una década más tarde, a un primer compromiso mundial acerca de seis objetivos educativos a alcanzar en 2015, que comprendían: atención y educación de la primera infancia (objetivo 1), enseñanza primaria universal (objetivo 2), las competencias de jóvenes y adultos (objetivo 3), la alfabetización de las personas adultas (objetivo 4), la igualdad de género (objetivo 5), y la calidad de la educación (objetivo 6).

También en el año 2000, los estados miembros de la Organización de las Naciones Unidas, y decenas de organizaciones internacionales, adoptaron la Declaración del Milenio, comprometiéndose así a respaldar el logro de los Objetivos de Desarrollo del Milenio, o sea, ocho puntos de referencia para el desarrollo internacional hasta 2015: erradicar la pobreza extrema y el hambre (objetivo 1), alcanzar la enseñanza primaria universal (objetivo 2), promover la igualdad de género y el empoderamiento de la mujer (objetivo 3), reducir la mortalidad de los niños menores de 5 años (objetivo 4), mejorar la salud maternal (objetivo 5), combatir el vih/sida, el paludismo y otras enfermedades (objetivo 6), garantizar la sostenibilidad del medio ambiente (objetivo 7), y fomentar una alianza mundial para el desarrollo (objetivo 8). Precisamente dentro de este marco mundial para el desarrollo, los objetivos de la Educación para Todos se consideraron 
como medios para alcanzar mejoras colectivas más amplias en términos de medios de vida, salud y crecimiento sostenible de las personas y los países.

Mientras, en 1997, la expansión industrial en Occidente, y el desarrollo económico en las emergentes economías asiáticas, habían llevado el desarrollo ecológicamente sostenible al primer plano también de la quinta CONFINTEA (UNESCO, 1997). En la última CONFINTEA en 2009 se han abordado las preocupaciones suscitadas no solo por la crisis financiera mundial recién comenzada en ese momento, sino también por los bajos niveles de logro alcanzados en los esfuerzos para reducir la brecha en la alfabetización de personas adultas, aumentar la integración social y asegurar los beneficios sociales de la educación (UNESCO, 2009).

En 2015, los gobiernos de los países miembros de la ONU firmaron la Agenda 2030 para el Desarrollo Sostenible que abarca diecisiete Objetivos que actualizan los de Desarrollo del Milenio que les precedieron, sobre un conjunto de temas que van desde la lucha contra la pobreza, el hambre y la erradicación hasta la lucha contra el cambio climático. Entre ellos, el objetivo 4 hace referencia a "garantizar una educación inclusiva, equitativa y de calidad y promover oportunidades de aprendizaje durante toda la vida para todos"; pero la educación también se relaciona con otros objetivos sobre salud y bienestar (objetivo 3), igualdad de género (objetivo 5), trabajo decente y crecimiento económico (objetivo 8), producción y consumo responsable (objetivo 12), y acción por el clima (objetivo 13).

En comparación con los objetivos del Milenio sobre educación que lo precedieron, aunque permanezcan los principios fundamentales en que se fundaba, o sea que la educación es un derecho fundamental y un bien público, también se distingue de ellos por su alcance geográfico, en cuanto no se limita a los países de bajos ingresos o en conflicto sino que se aplica a todos lo países, independientemente de sus niveles de ingresos o desarrollo. Además incluye metas con un enfoque sobre todas las personas vulnerables (meta 4.5) y también sobre jóvenes y adultos y sus competencias básicas de cálculo numérico, lectura y escritura (meta 4.6) y para el trabajo (meta 4.4). Por eso, en el Congreso de Revisión a medio plazo de la sexta CONFINTEA que se celebró en octubre de 2017 se ha debatido sobre asuntos como: ¿Cuáles deberían ser las prioridades de los países también considerando el rol de apoyo de la educación y el aprendizaje de personas adultas en el logro de los Objetivos de Desarrollo Sostenible? (UIL, 2017).

Resumiendo, lo que destaca de estas anotaciones históricas es que la institucionalización de la cooperación entre países que se produjo en la posguerra de la Segunda Guerra Mundial con el establecimiento de la UNESCO, entre otras organizaciones internacionales, ayudó a convertir los intercambios de conocimientos e ideas sobre la educación entre las personas, en intercambios entre organizaciones como modalidad predominante de gobernanza mundial. Durante este mismo período, la institucionalización 
de la cooperación entre los gobiernos ha creado las condiciones para la adaptación de unos instrumentos de política internacional que se han aplicado cada vez más para gobernar y dirigir la política educativa a nivel mundial, y de lo que tanto los congresos cuanto los compromisos sobre objetivos internacionales son ejemplos.

Merece también destacar que, siendo la educación un campo de jurisdicción interna de los países, para dirigir la política educativa a nivel mundial la UNESCO solo puede utilizar instrumentos jurídicos, como son sus recomendaciones, bastante 'blandos', ya que alientan a los gobiernos a adoptar ciertos enfoques o emprender determinadas actuaciones, pero no son jurídicamente vinculantes. También así sus recomendaciones contribuyen al ejercicio de un gobierno tanto normativo cuanto cognitivo que, por un lado, despliega conocimiento e ideas convergentes sobre la educación, y, por otro lado, favorece la ingeniería y la propagación de un conjunto de valores, perspectivas, expectativas y discursos sobre la educación y su papel para el desarrollo de las personas y los países. Por eso, la labor normativa en la EPA así como el trabajo conceptual sobre la educación como bien común, desplegados en estos últimos años por la UNESCO, merecen una atención particular para entender las transformaciones acaecidas en el campo durante los últimos años.

\section{La labor normativa: La Recomendación sobre el aprendizaje y la educa- ción de adultos de 2015}

La labor normativa de la UNESCO en el campo de la educación de adultos tiene sus raíces en la tercera CONFINTEA 1972, cuando los Estados miembros autorizaron a la Dirección General de la UNESCO a realizar este trabajo que derivó en la aprobación de la primera Recomendación sobre el desarrollo de la educación de adultos de 1976. Como ya he señalado, las recomendaciones son el más suave de los instrumentos jurídicos a nivel internacional. Esta Recomendación no es una excepción. Aún así, como instrumento normativo que fomenta la conformidad con determinadas normas sociales y establece niveles deseables de rendimiento, la Recomendación contribuyó a estimular el debate político sobre la EPA, al menos en los países de bajo ingresos. De hecho, proporcionó una definición exhaustiva de la educación de personas adultas y estableció unos principios para asegurar su provisión. Lo que merece destacarse es que la EPA se veía, por primera vez, reconocida a nivel mundial como "un aspecto fundamental del derecho a la educación" (Recomendación sobre el desarrollo de la educación de adultos de 1976, p.1), y por lo tanto un derecho humano universal, que otorga reconocimiento a las experiencias de los individuos y apoya la integración "de los grupos educativamente más desfavorecidos en todos los niveles de la vida comunitaria" (Recomendación sobre el desarrollo de la educación de adultos de 1976, p.5). 
Al comenzar la crisis económica mundial, o sea a partir de 2009, se puso en marcha un proceso de revisión y actualización de esta recomendación bajo la coordinación del Instituto de la UNESCO para el Aprendizaje Permanente que acabó en la adopción por la Conferencia General de la UNESCO de la Recomendación sobre el aprendizaje y la educación de adultos de 2015. Esta nueva Recomendación, que reemplaza la previa, proporciona una definición muy amplia de lo que comprende el aprendizaje y la educación de personas adultas y su alcance. Esto incluye

también las posibilidades de educación y aprendizaje para la ciudadanía activa conocidas como educación comunitaria, popular o liberal... [En cuanto] Faculta a las personas para participar activamente en la solución de problemas sociales como la pobreza, las cuestiones de género, la solidaridad intergeneracional, la movilidad social, la justicia, la equidad, la exclusión, la violencia, el desempleo, la protección ambiental y el cambio climático. Ayuda también a las personas a llevar una vida digna desde el punto de vista de la salud y el bienestar, la cultura, la espiritualidad y en relación con todos los demás ámbitos que contribuyen al desarrollo y la dignidad personales (artículo 6).

La Recomendación también menciona entre las metas del aprendizaje y la EPA la de

dotar a las personas de las capacidades necesarias para ejercer sus derechos y hacerlos efectivos, y hacerse cargo de su propio destino (artículo 8), con los objetivos, entre otros, de ... contribuir a crear una sociedad ... en la que cada individuo tenga la oportunidad de aprender y participar con plenitud en procesos de desarrollo sostenible, y aumentar la solidaridad de las personas y las comunidades (artículo 9).

Con tal fin se señalan como ámbitos de acción los que ya se habían identificado en el Marco de acción de Belém aprobado por la sexta CONFINTEA, o sea: las políticas, la gobernanza, la financiación, la participación, inclusión y equidad, y la calidad del aprendizaje y la educación de adultos.

Lo que aquí merece destacarse es el ámbito de la participación, inclusión y equidad y su referencia a que

Se debería prestar particular atención a determinados grupos destinatarios con objeto de reconocer su contribución al desarrollo social, respetando al mismo tiempo la diversidad cultural y otras formas de diversidad... [y] no tolerar ningún tipo de discriminación por ningún motivo (artículo 23). 


\section{El trabajo conceptual: Replantear la educación ¿Hacia un bien común mundial? de 2015}

El labor conceptual que también estoy interesada en resaltar tiene sus raíces en 1972, y por lo tanto se ha desarrollado paralelamente a la labor normativa que acabo de mencionar. Sus primeras semillas pueden encontrarse en el informe de la Comisión Internacional del Desarrollo de la Educación coordinada por Edgar Fauré: Aprender a ser: la educación el futuro (1972).

Este encarna el trabajo que se había llevado a cabo en torno al concepto de "educación permanente" bajo el auspicio del Instituto de Educación de la UNESCO en un momento de liderazgo activo en el establecimiento del principio organizador del desarrollo educativo (Tuijnman y Boström, 2002). Fue reconocido por el "movimiento de educación permanente" (Wain, 2001, p. 184) por su enfoque radical, que también conectaba con los movimientos a favor de la desescolarización y desinstitucionalización de la educación (Moosung y Friedrich, 2011). En resumen, el informe revela que la noción de educación permanente hacía referencia primaria a la necesidad de crear nuevas y diversas oportunidades de educación y aprendizaje para ampliar procesos democráticos, dentro de un proyecto radical que replanteaba la naturaleza misma de la educación y la cultura como procesos que "trascienden los límites de las instituciones, los programas y los métodos impuestos a lo largo de los siglos" (Faure et al., 1972, p.145). Este proyecto fue fundamentado en ideas liberales socialdemócratas que vieron el crecimiento individual como inevitablemente vinculado con el desarrollo social (Milana, 2012).

A mediados de la década de mil novecientos noventa, cuando los argumentos del capital humano impregnaban las preocupaciones mundiales sobre el crecimiento económico, un Comité de la UNESCO, bajo la coordinación de Jacques Delors, publicó otro informe, La educación encierra un tesoro (1996), en que las ideas sobre la educación inspiradas en el capital humano se contrarrestaron en cierto modo por un enfoque socialdemócrata liberal. Por lo tanto, basándose en las conexiones entre el crecimiento económico de las naciones, las preocupaciones por la equidad y la preocupación por el medio ambiente, el informe destacó el papel del estado de bienestar, e hizo que la educación recuperara una posición central en las agendas políticas mundiales.

Aunque el informe no sea inmune a las ideas neoliberales, como la actualización de las habilidades personales, representa un intento de preservar un enfoque socialdemócrata liberal en la educación, que reconcilia crecimiento económico con problemas de equidad y respeto por la condición humana y el medio ambiente. Por lo tanto reafirma el papel central del estado de bienestar en un momento histórico en que ya iba siendo seriamente cuestionado por la expansión del pensamiento neoliberal en educación (Milana, 2012; Moosung y Friedrich, 2011). 
Ambos informes han inspirado las políticas internacionales de educación en los decenios de 1970, y de nuevo a partir de 1990 (Tawil y Cougoureux, 2013, Elfert, 2015). En años recientes estos mismos informes han sido tomados como puntos de partida por el trabajo conceptual que se ha concretizado en el documento Replantear la educación ¿Hacia un bien común mundial? de 2015, y que se publicó en una coyuntura histórica particular, o sea cuando la comunidad internacional de educación y desarrollo estaba aprobando la ya mencionada Agenda 2030 para el Desarrollo Sostenible. De hecho, Replantear la educación es un documento regulador y ha sido fuente de inspiración para el Marco de Acción también conocido como Educación 2030 para la realización del Objetivo de Desarrollo Sostenible cuatro sobre la educación.

En Replantear la educación se exponen una serie de tendencias y tensiones en un mundo caracterizado por el incremento de la complejidad, la incertidumbre y las contradicciones, y por eso se sugiere que el propósito de la educación y de la organización del aprendizaje sea revisado a partir de un enfoque humanista.

En síntesis se plantea que el principio de la educación como un bien público está bajo tensión debido al aumento dramático de los actores no estatales en la educación, y por eso también a los riesgos relacionados con su cosificación y mercantilización. En particular, con el concepto de 'bien común' se hace referencia a todos aquellos "bienes que los seres humanos comparten intrínsecamente en común y que se comunican entre sí, como los valores, las virtudes cívicas y el sentido de la justicia" (Deneulin y Townsend, 2007, p. 142). Por lo tanto son bienes comunes los que se realizan "en las relaciones reciprocas en las cuales y por medio de las cuales los seres humanos consiguen su bienestar" (Cahill citado en Deneulin y Townsend, 2007, p. 143). Por extensión, entender la educación como un bien común permite, según los autores de Replantear la educación, sobrepasar una concepción instrumental de la educación, para reconocer la diversidad de interpretaciones que, en diferentes contextos, cosmovisiones y sistemas de conocimiento, definen lo que constituye un bien común, y el carácter participativo de los procesos a través de los cuales se desarrolla la educación como acción común. Por lo tanto Replantear la educación plantea la noción de la educación como 'bien común' para contrarrestar los discursos hegemónicos de la educación basada en los principios del capital humano y del racionalismo económico, a fin de re-contextualizar el conocimiento y la educación como derechos humanos, pero también para re-contextualizar el gobierno de ambos. Aún así el documento deja muchas preguntas por responder sobre cómo poner en práctica este paradigma, entre ellas: ¿Cómo reafirmar la dimensión colectiva de la educación en cuanto esfuerzo social compartido? ¿Cómo alcanzar y mantener un núcleo común de valores compartidos? y ¿Cómo reconocer e integrar la diversidad de conocimiento, contextos y conceptos de bienestar en la educación? 


\section{Implicaciones para la educación de personas adultas}

En resumen, tanto la labor normativa sobre la educación de personas adultas cuanto el trabajo conceptual sobre la educación como bien común realizado por la UNES$\mathrm{CO}$, tienen el objetivo último de empoderar a los seres humanos y a sus relaciones reciprocas, y por eso tienen implicaciones para la EPA. Las dimensiones ideológicas en que se fundan incluyen una interpretación holística y humanista del desarrollo social y un enfoque integrado para conseguir el bienestar de los seres humanos.

La interpretación holística del desarrollo social reconoce la interdependencia entre todas las propiedades de un actor o estructura social, así como la interdependencia entre la autorrealización individual y la formación de identidades colectivas y de la sociedad en general. Esta creencia está en agudo contraste con el impulso neoliberal hacia una sociedad aún más individualista, caracterizada por la independencia y la autosuficiencia, donde se cree, erróneamente, que las acciones sociales sean moralmente independientes, autónomas, esencialmente no sociales (Dumont, 1986). Una tendencia que, aunque observada en las sociedades contemporáneas, no representa un determinismo social que no pueda ser desafiado.

La preocupación humanista por un tipo de desarrollo social concibe los seres humanos como 'verdaderamente humanos' al mejorar las formas de vida autónomas en una sociedad caracterizada por la injusticia social y las relaciones desiguales. Esto implica una preocupación especial por el aumento de la capacidad individual y colectiva para enfrentar la vida. De acuerdo con esta convicción, la atención de la EPA tiene que reorientarse a las necesidades de los seres humanos en lugar de a sus déficits. De alguna forma, podríamos encuadrarla más como un proyecto para el desarrollo social, y no como una respuesta directa a las disfunciones y déficits sociales y educativos.

El enfoque integrado para la mejora de la calidad de vida no puede relegarse a una etapa específica de la vida, ni a una de las muchas dimensiones de la vida. Eso impone que la EPA no solo sea aplicable en varios servicios y actividades fuera de la escuela y la familia, sino también para todos los grupos de edad. En este sentido, abarca "patrones de aprendizaje formales, no formales e informales a lo largo del ciclo de vida de un individuo para el mejoramiento consciente y continuo de la calidad de vida individual y de la sociedad" (Dave citado en Medel-Añonuevo et al., 2001, p. 2).

Para recapitular, las políticas sociales y educativas han adoptado tradicionalmente un enfoque de compensación de déficits. El déficit ha sido interpretado de manera diferente como un riesgo para la sociedad o como un problema de movilización de recursos en lugar de su redistribución. Es decir, que las políticas sociales y educativas se han centrado principalmente en encontrar formas para proporcionar algún tipo de compensación a nivel individual. En consecuencia, la EPA se ha entendido, desde ciertos enfoques, como una respuesta a la desigualdad social y la injusticia que resultan 
de los procesos de desintegración, jerarquía de género, conflictos étnicos, etc. El cambio ideológico explicado anteriormente, sin embargo, sugiere una agenda social más amplia, que implica alejarse de considerar la EPA como una práctica compensatoria individual hacia su reinterpretación como una combinación de educación no formal y aprendizaje para el derecho a la ciudadanía. Y eso implica, como había adelantado al principio de estas páginas, la necesidad, por un lado, de seguir reflexionando sobre la idea de educación como bien común, a pesar de sus criticidades, y por otro, de seguir actuando a favor de un desarrollo sustentable que rechace el modelo antropocéntrico de sostenibilidad de matriz economicista.

En resumen, con el apoyo de la UNESCO y organizaciones no gubernamentales como el Consejo Internacional para la Educación de Adultos (ICAE), los acuerdos internacionales han hecho visible que la inversión pública en EPA no sea sólo una prerrogativa para pocos, sino necesaria en todos los países, independientemente del producto interior bruto nacional. Sin embargo, también se destaca en el Tercer Informe Global sobre Aprendizaje y Educación de Adultos (UIL, 2016) que, a pesar de la creciente evidencia de que la EPA brinde múltiples beneficios (en términos de salud y bienestar, empleo y mercado de trabajo, vida social, cívica y comunitaria), una mirada a las realidades nacionales, pone en evidencia que estas parecen todavía poco sensibles a los cambios políticos e ideológicos recién delineados. Por lo tanto, existe una gran necesidad de que los educadores y educadoras y los investigadores e investigadoras sensibilicen a otras instituciones sociales y respalden estas transformaciones en su trabajo.

\section{Notas}

1 Este texto se basa en la conferencia impartida en el XXX Seminario Interuniversitario de Pedagogía Social: "Pedagogía Social y Desarrollo Humano" celebrado en Sevilla del 8 al 10 de noviembre 2017

\section{Referencias}

Delors, J., Al Mufti, I.a., Amagi, I., Carneiro, R., Chung, F., Geremek, B., ..., y Nanzhao, Z. (1996). La educación encierra un tesoro - Informe a la UNESCO de la Comisión Internacional sobre la Educación. Paris: UNESCO.

Deneulin, S., y Townsend, N. (2007). Public Goods, Global Public Goods and the Common Good. International Journal of Social Economics, 34 (1-2), 19-36.

Dumont (1986). Essays on individualism: Modern ideology in anthropological perspective. Chicago/ London: University of Chicago Press.

Elfert, M. (2015). UNESCO, the Faure report, the Delors report, and the political utopia of lifelong learning. European Journal of Education, 50(1), 88-100.

Faure, E., Herrera, F., Kaddoura, A.-R., Lopes, H., Petrovsky, A. V., Rahnema, M., y Ward, F. C. (1972). Aprender a ser: La educación del futuro. Paris: UNESCO. 
Freire, P. (1970). Pedagogía del Oprimido. Montevideo: Tierra Nueva.

Knoll, J. H. (2007). The history of the UNESCO International Conferences on Adult Education. Convergence, 40(3/4), 21-41.

Lengrand, P. (1970). Una Introducción a la Educación Permanente. Paris: UNESCO.

Lima, L. C. (2017). Adult education in times of crisis: The contributions of Paulo Freire and Ettore Gelpi to policy studies. Conferencia pronunciada en el Tercer Congreso de la red de investigación sobre Políticas de Educación de Adultos de la la Sociedad Europea para la Investigación en Educación de Adultos (ESREA).

Medel-Añonuevo, C., Ohsako, T. \& Mauch, W. (2001). Revisiting Lifelong Learning for the 21st Century. Hamburg: UNESCO Institute for Education.

Milana, M. (2012). Political globalization and the shift from adult education to lifelong learning. The European Journal for Research on the Education and Learning of Adults, 3(2), 103-117.

Moosung, L., y Friedrich, T. (2011). Continuously reaffirmed, subtly accommodated, obviously missing and fallaciously critiqued: ideologies in UNESCO's lifelong learning policy. International Journal of Lifelong Education, 30(2), 151-169.

Recomendación sobre el aprendizaje y la educación de adultos del 13 de noviembre de 2015 por la Organización de las Naciones Unidas para la Educación, la Ciencia y la Cultura.

Recomendación sobre el desarrollo de la educación de adultos 26 de octubre al 30 de noviembre de 1976 por la Organización de las Naciones Unidas para la Educación, la Ciencia y la Cultura.

Tawil, S. y Cougoureux, M. (2013). Revisiting Learning: The treasure within - Assessing the in uence of the 1996 Delors report. Paris: UNESCO Education Research and Foresight, ERF Occasional Papers, No. 4.

Tuijnman, A. C., y Boström, A.-K. (2002). Changing Notions of Lifelong Education and Lifelong Learning. International Review of Education, 48(1/2), 93-110.

UNESCO. (1997). The Hamburg declaration - The Agenda for the future. Fifth International Conference on Adult Education 14-18 July. Hamburg: UNESCO Institute for Education.

UNESCO. (2009). Harnessing the power and potential of adult learning and education for a viable future - Bélém Framework for Action. CONFINTEA VI Bélém, 4 December. Hamburg: UNESCO Institute for Lifelong Learning.

UNESCO (2015). Replantear la educación ¿Hacia un bien común mundial? Paris: UNESCO.

UNESCO, MEC. (2014). Adult education in retrospective: 60 years of CONFINTEA. Brasilia: UNESCO, MEC.

UIL. (2016) Third Global Report on Adult Learning and Education: The Impact of Adult Learning and Education on Health and Well-Being; Employment and the Labour Market; and Social, Civic and Community Life. Hamburg: UNESCO Institute for Lifelong Learning.

UIL. (2017). CONFINTEA VI Mid-Term Review, 25-27 October 2017 Suwon, Republic of Korea - Report of the conference. Hamburg: UNESCO Institute for Lifelong Learning. 
Wain, K. (2001). Lifelong Learning: Small Adjustments or Paradigmatic Shift? In D. Aspin, J. Chapman, M. Hatton, y Y. Sawano (Eds.), International Handbook of Lifelong Learning (Vol. I, pp. 183-198). Dordrecht: Kluwer Academic Publishers.

Marcella Milana

Profesora asociada en la Universidad de Verona, Italia, donde dirige el International Research Centre for Global and Comparative Policy Studies on the Education and Learning of Adults (IRC-GloCoPos), y se dedica a la investigación de la política y la gobernanza de la educación de personas adultas, desde perspectivas comparativas y mundial.

Email: marcella.milana@univr.it ORCID ID: 0000-0002-3068-3530

Correspondencia:

Dr. Marcella Milana

Università di Verona, Dipartimento di Scienze Umane Lungadige Porta Vittoria, 17 37129 Verona, Italia

Data de Submissão: Janeiro 2018

Data de Avaliação: Abril 2018 Data de Publicação: Dezembro 2018 